\title{
RHINOLOGY
}

\section{Association of gastro-oesophageal reflux disease and quality of life in patients with chronic rhinosinusitis}

\section{Associazione della malattia da reflusso gastroesofageo e la qualità di vita dei pazienti affetti da rinosinusite cronica}

\author{
Yang $\mathrm{Xu}^{1,2}$, Wen Yang ${ }^{1}$, Yao Song ${ }^{1}$, Danni Cheng ${ }^{1}$, Min $\mathrm{CaO}^{3}$, Jingyue Huang ${ }^{4}$, Jianjun Ren ${ }^{1,2}$, Jong Wook Lee

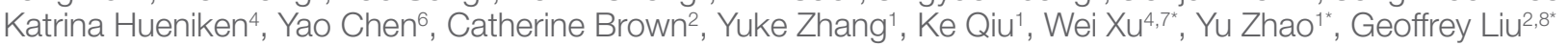 \\ ${ }^{1}$ Department of Otorhinolaryngology Head and Neck Surgery, West China Hospital, West China Medical School, Sichuan University, \\ Chengdu, Sichuan, China; ${ }^{2}$ Medical Oncology and Medical Biophysics, Princess Margaret Cancer Centre, University of Toronto, \\ Toronto, Ontario, Canada; ${ }^{3}$ General Affairs Office of Logistic Department, West China Hospital, Sichuan University, Chengdu, \\ Sichuan, China; ${ }^{4}$ Department of Biostatistics, Princess Margaret Cancer Centre, Toronto, Ontario, Canada; ${ }^{5}$ Department of \\ Otolaryngology Head and Neck Surgery, University of Toronto, Toronto, Ontario, Canada; ${ }^{6}$ Department of Gastroenterology, \\ Chengdu First People's Hospital, Chengdu, Sichuan, China; 7 Dalla Lana School of Public Health, University of Toronto, Toronto, \\ Ontario, Canada; ${ }^{8}$ Medicine and Epidemiology Dalla Lana School of Public Health, University of Toronto, Toronto, Ontario, Canada \\ G. Liu, Y. Zhao and W. Xu contributed equally to this work.
}

\section{SUMMARY}

Objective. We determined the influence of gastro-oesophageal reflux disease (GERD) on quality of life (QOL) before and after functional-endoscopic-sinus-surgery (FESS) for chronic rhinosinusitis (CRS).

Methods. Medically-recalcitrant CRS patients were recruited prior to FESS. GERD was diagnosed endoscopically. QOL was compared between patients with $v s$ without GERD at baseline and one-year post-FESS.

Results. Of 187 CRS patients receiving FESS, 40 had GERD. Pre-operative QOL was significantly worse in CRS patients with $v s$ without GERD. Pre-operative GERD healthrelated QOL (GERD-HRQL) and reflux symptom index (RSI) scores were both correlated with pre-operative SNOT-22 scores. Compared with non-GERD CRS patients, GERD patients demonstrated larger SNOT-22 improvements after FESS, such that post-operative SNOT-22 values were no longer significantly different between GERD and non-GERD groups. However, post-FESS, in patients with CRS without nasal polyps (unlike those with nasal polyps), the GERD ( $v s$ non-GERD) group suffered from greater sleep dysfunction and otologic/facial symptoms.

Conclusions. Compared to CRS patients without GERD, those with GERD experienced poorer pre-operative QOL and greater QOL improvement after FESS.

KEY WORDS: chronic rhinosinusitis, gastroesophageal reflux disease, functional endoscopic sinus surgery, proton pump inhibitors, health-related quality of life

\section{RIASSUNTO}

Obiettivo. Abbiamo determinato l'impatto della malattia da reflusso gastroesofageo $(G E R D)$ sulla qualità della vita (QOL) prima e dopo chirurgia endoscopica funzionale nasosinusale (FESS) nel trattamento della rinosinusite cronica (CRS).

Metodi. I pazienti con CRS refrattari alle terapie mediche sono stati reclutati prima della FESS. La diagnosi di GERD è stata raggiunta endoscopicamente. La qualità della vita è stata confrontata tra pazienti con e senza GERD al basale e a un anno dopo la FESS.

Risultati. Dei 187 pazienti con CRS sottoposti a FESS, 40 erano affetti da GERD. La qualità della vita prima dell'intervento era significativamente peggiore nei pazienti affetti da CRS e con GERD rispetto a quelli senza GERD. I punteggi preoperatori della qualità della vita in relazione alla GERD (GERD-HRQL) e dell'indice dei sintomi da reflusso (RSI) sono stati correlati ciascuno con i punteggi SNOT-22 preoperatori. Rispetto ai pazienti con CRS senza GERD, i pazienti con GERD hanno dimostrato miglioramenti della SNOT-22 più significativi dopo trattamento FESS, $i$ valori SNOT-22 postoperatori non erano più significativamente differenti tra i gruppi con GERD e senza GERD. Tuttavia, dopo la FESS, nei
Received: February 18, 2021

Accepted: July 29, 2021

\section{Correspondence}

Geoffrey Liu

Medical Oncology and Medical Biophysics, Princess Margaret Cancer Centre, University of Toronto, Toronto, Ontario, Canada; Medicine and Epidemiology Dalla Lana School of Public Health, University of Toronto, Toronto, Ontario, Canada E-mail: Geoffrey.Liu@uhn.ca

\section{Funding}

This study was funded by International Cooperation Project of Science and Technology Department of Chengdu City (Grant Number: 2018-YFYF-00123$\mathrm{SN}$ ), Fundamental Research Funds for the Central Universities (Grant Number: 012017yjsy118), 1-3-5 Project for Disciplines of Excellence-Clinical Research Incubation Project of West China Hospital of Sichuan University (Grant Number: 2019HXFH003).

\section{Conflict of interest}

The Authors declare no conflict of interest.

How to cite this article: Xu Y, Yang W, Song Y, et al. Association of gastro-oesophageal reflux disease and quality of life in patients with chronic rhinosinusitis. Acta Otorhinolaryngol Ital 2021;41:450460. https://doi.org/10.14639/0392-100X-N1491

(C) Società Italiana di Otorinolaringoiatria e Chirurgia Cervico-Facciale

\section{c) $(\mathbf{P})$}

This is an open access article distributed in accordance with the CC-BY-NC-ND (Creative Commons Attribution-NonCommercial-NoDerivatives 4.0 International) license. The article can be used by giving appropriate credit and mentioning the license, but only for non-commercial purposes and only in the original version. For further information: https:// creativecommons.org/licenses/by-nc-nd/4.0/deed.en 
pazienti con CRS senza poliposi nasale (a differenza di quelli con poliposi nasale), il gruppo GERD (contro non-GERD) ha avuto una maggiore disfunzione del sonno e sintomi otologici/facciali.

Conclusioni. Rispetto ai pazienti con CRS senza GERD, quelli con GERD hanno beneficiato di un miglioramento della QOL maggiore dopo trattamento FESS.

PAROLE CHIAVE: rinosinusite cronica, malattia da reflusso gastroesofageo, chirurgia endoscopica funzionale dei seni paranasali, inibitori della pompa protonica, qualità della vita correlata alla salute

\section{Introduction}

Chronic rhinosinusitis (CRS) is a common inflammatory condition of the nose and paranasal sinuses, with significant impact on quality of life (QOL) and productivity ${ }^{1}$. In mainland China, the estimated prevalence of CRS is $8 \%$ with worldwide rates ranging from $5.5 \%$ to $10.9 \%{ }^{2}$. Patients with CRS use $152 \%$ greater healthcare services and produce $139 \%$ higher healthcare costs than age-matched controls in the Chinese population ${ }^{3}$.

Gastro-oesophageal reflux disease (GERD) is one of the most commonly encountered conditions by primary care providers and is frequently associated with extra-oesophageal conditions including dental erosions, chronic laryngitis and laryngopharyngeal reflux (LPR). The prevalence of GERD in China is approximately $3.8 \%$ with a worldwide increasing trend in the past two decades ${ }^{4}$. Like CRS, GERD is associated with lower work productivity and increased health utilisation in those with severe and frequent symptoms.

Growing evidence has implicated a role of GERD in CRS. A recent meta-analysis of epidemiological studies reported a significant positive association between the two conditions ${ }^{5}$. CRS patients with GERD are shown to have worse sinusitis related-QOL compared to CRS patients without GERD.

Functional endoscopic sinus surgery (FESS) is a minimally invasive technique to recover normal sinus function and widely used in CRS patients after failure of drug treatment ${ }^{1}$. Studies have reported that FESS results in symptomatic improvement in 76-98\% of CRS patients. However, few studies have investigated the prognostic influence of GERD on outcomes of FESS, and there is no specific study evaluating QOL after initial FESS stratified by the presence or absence of GERD.

Relevant studies have yielded mixed results. Some studies report a higher prevalence of GERD in patients with recalcitrant CRS following FESS compared to those with resolved symptoms ${ }^{6,7}$. A retrospective study by Chambers et al. that collected key pre-operative variables at a mean of 42.5 months after surgery contains potential recall bias ${ }^{6}$. Confounders including comorbidities were not controlled in DelGaudio's study ${ }^{7}$. Another study found no difference in symptoms and endoscopic outcomes before and after FESS between those with or without GERD, but used prior self-reported history or anti-reflux medication as a proxy definition ${ }^{8}$.
The objective of our study is to evaluate the relationship between the presence or absence of a concurrent confirmed GERD diagnosis in CRS patients with pre- and post-FESS outcomes.

\section{Materials and methods}

This prospective cohort study was approved by the Biomedical Research Ethics Committee of West China Hospital, Sichuan University (2018 No.65), with informed consent obtained from all patients.

Research involved human participants only. All procedures performed involving human participants were in accordance with the ethical standards of the institutional and national research committee and with the 1964 Helsinki declaration and its later amendments or comparable ethical standards. Informed consent was obtained from all individual participants included in the study.

\section{Population}

Adults diagnosed with CRS were recruited at the West China Hospital between 2016 and 2018. All participants lived in Southwestern China, mainly Sichuan Province. Eligible patients met the clinical CRS definition with symptoms over 12 weeks in duration, along with computed tomography (CT) and endoscopic image documentation of CRS. Subjects had to agree to FESS after medical management was unsuccessful in alleviating symptoms. Patients with acute rhinosinusitis, fungal rhinosinusitis and cilia dyskinesia were excluded. Patients who failed to complete the initial clinical interview or one-year post-FESS follow up were excluded from the analysis. Patients receiving longterm oral corticosteroid or nonsteroidal agents for anti-inflammatory purposes other than for CRS were also excluded. CRS was confirmed secondarily at the time of FESS through histological analysis of the resected tissue.

\section{Diagnosis and management of GERD}

Our institutional protocol for the diagnosis and management of GERD at the gastroenterology department is shown in Figure 1. All study subjects were assessed for GERD prior to surgery following the recruitment of this study.

After completion of the epidemiologic questionnaire with CRS and GERD related QOL evaluation, all patients re- 


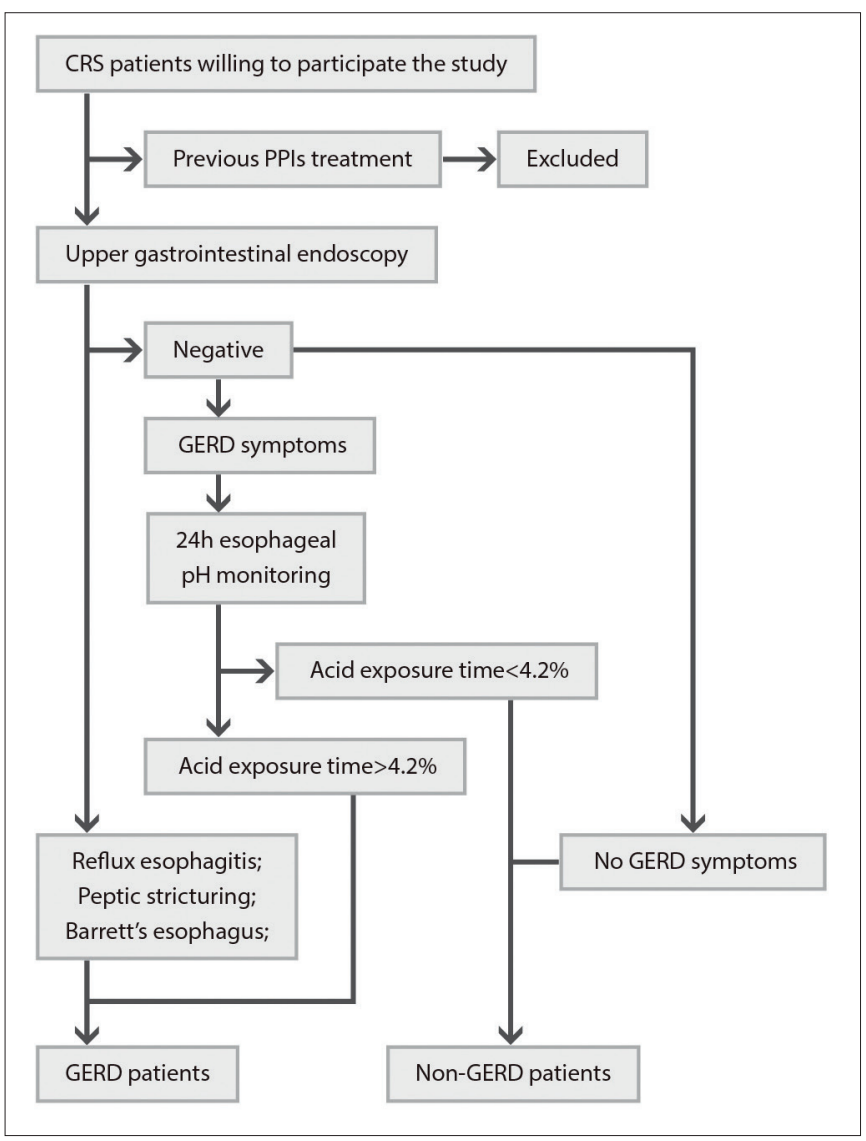

Figure 1. Flow diagram of how patients with chronic rhinosinusitis (CRS) were assessed for gastro-oesophageal reflux disease (GERD).

ceived an upper gastrointestinal endoscopy at the gastroenterology department to detect signs of GERD, those with reflux oesophagitis, peptic stricturing, or Barrett's oesophagus were grouped into subpopulation with GERD. GERD symptoms were inquired among patients of negative results from upper gastrointestinal endoscopy, including typical symptoms (regurgitation and heartburn) and extra-oesophageal symptoms (e.g. chronic throat clearing or hoarseness). Twenty-four-hour $\mathrm{pH}$ monitoring was performed to subjects with GERD symptoms, with acid exposure time $(\mathrm{AET})>4.2 \%$ defined as GERD. CRS patients without GERD symptoms and signs, or with AET $<4.2 \%$ were classified as non-GERD patients ${ }^{9}$.

The four patients who had been treated with proton pump inhibitors (PPIs) prior to participating in this study were excluded due to heterogeneity and lack of previous supportive documents of GERD diagnosis. The patients who failed to complete the therapy for GERD at the time of follow-up were excluded. Also excluded from analyses were the rare patients whose testing led to anti-reflux surgery or use of baclofen for oesophageal spasms, patients without GERD but receiving anti-reflux PPIs treatment for other reasons, and the few with refractory GERD-like symptoms but caused by other aetiologies (e.g. cardiac chest pain) after completing the extensive work-up (total of five patients)..$^{10}$ The pathogenesis of eosinophilic oesophagitis (EoE) is distinct from GERD, and EoE has been linked to CRS ${ }^{11}$. We excluded EoE patients in our study.

GERD patients were given PPIs treatment. PPIs switching, titration (e.g. doubling of dose) and compliance-assessment were used to optimise medical management of GERD ${ }^{10}$.

\section{Data collection}

An epidemiologic questionnaire collected data on age, sex, body mass index (BMI), home address, ethnicity, smoking, alcohol use and previous medical history at enrollment. The phenotypes of CRS were recorded: CRS with nasal polyp (CRSwNP) and CRS without nasal polyp (CRSsNP) ${ }^{1}$. Comorbidities were collected through hospital records, including asthma, allergic rhinitis (AR), deviated septum, obstructive sleep apnoea hypopnoea syndrome (OSAHS), diabetes and hypertension. The guidelines recommended by the Global Initiative for Asthma was used to define the diagnosis of asthma ${ }^{12}$. AR diagnosis was based on concordance between atopy test results and a typical history of allergic symptoms ${ }^{13}$. Smoking status was divided into previous smokers, current smokers and never smoker. Alcohol consumption was dichotomised into less than twice a week and twice a week or more.

\section{FESS procedures}

FESS was performed under general anaesthesia by the same set of surgeons, independent of GERD status. Surgery utilised mechanical instruments with a microdebrider to minimise damage to nasal mucosa. Surgery involved removal of polyps and diseased mucosa with opening of the next anatomical compartment. The extent of the procedure was tailored to individual patients, if necessary, the procedure included opening of the ethmoidal, frontal and/ or sphenoidal sinuses. The surgical technique has been described by Stammberger and Kennedy ${ }^{14,15}$.

\section{Post FESS management}

All patients undergoing FESS were treated post-operatively with 2-weeks of antibiotics and nasal corticosteroids. ${ }^{1}$ Intranasal corticosteroids were used to alleviate post-operative symptoms. The Nasopore packing was used to control post-operative bleeding ${ }^{16}$. Routine nasal endoscopic follow-up was at 2, 4, and 12 weeks after FESS.

\section{QOL evaluation and objective testing}

QOL evaluations were conducted pre-operatively prior to GERD assessment or treatment. Post-operative QOL was 
evaluated at one-year follow up after FESS. The Sinonasal Outcome Test-item 22 (SNOT-22), used to assess sinonasal related QOL, is a validated self-reported 22-item questionnaire, assessed on a Likert scale from 0 (symptom absent) to 5 (worst symptom possible), divided into five subdomains: rhinologic symptoms, extranasal rhinologic symptoms, otologic/facial symptoms, psychological dysfunction, and sleep dysfunction ${ }^{17}$.

Gastroesophageal Reflux Disease-Health Related Quality of Life (GERD-HRQL), pre-operatively evaluated along with SNOT-22, is a validated 10-item method of measuring GERD symptom severity, ranging from 0 (asymptomatic in all items) to 50 (incapacitated in all items).

Reflux Symptom Index (RSI), also collected with pre-operative SNOT-22, is a validated 9-item, self-administered questionnaire designed to evaluate symptoms of extra-oesophageal reflux with each item ranging from 0 (absent) to 5 (severe) and a maximum score of 45 .

Sinus CT scan was evaluated through the 12-item LundMackay system pre-FESS, and used for radiologic staging of CRS; the system has 24 total points, with $0-2$ points given for each sinus group: 0 point (no abnormality), 1 point (partial opacification), and 2 points (complete opacification). Sinus involvement was defined as having a LundMackay score $\geq 1$.

Endoscopic results were assessed by the Lund-Kennedy system at baseline and 12 weeks post-FESS, using a 10 -item scoring system of five items indicating severity of polyp, discharge, oedema, crusting and scarring, with 0-2 points (higher scores $=$ severe CRS signs) on each side, for a maximum of 20 points.

\section{Statistical analysis}

Data was double-entered and quality control steps were performed using Epidata (Version 2.0). Demographic characteristics were presented descriptively. Changes in SNOT-22 scores from pre-operative to one-year post-operative assessments and subdomain scores were presented visually using boxplots. Spearman correlations assessed relationships between GERD-HRQL, RSI, and SNOT-22 scores, while Shapiro-Wilk test lacked normality of SNOT-22 and Lund-Mackay scores leading to quantile normalisation prior to linear regression on transformed scores. Associations between clinico-demographic variables including presence/absence of GERD were assessed using univariable and multivariable regression with outcomes of: preoperative SNOT-22 score; improvement in SNOT-22 score (defined as the difference between pre-operative and postoperative scores); and pre-operative Lund-Mackay score. A multivariable linear regression model was conducted with forward selection algorithm on plausible risk factors selected by clinical judgement, including sex, age, BMI and smoking status. Both SNOT-22 overall and subdomain scores (pre-operative, post-operative and difference between pre- and post-operative scores) were compared between GERD and non-GERD groups. Further subset analyses were conducted by the presence or absence of nasal polyps (e.g. CRSsNP patients; CRSwNP patients). Factors associated with post-operative QOL improvement in CRSsNP/CRSwNP patients were tested in univariable and multivariable analyses. We evaluated the CRS findings of different groups of sinuses involvement (frontal sinus, anterior ethmoid sinus, posterior ethmoid sinus, sphenoid sinus and maxillary sinus).

All statistical tests were two-sided with statistical significance defined as $\mathrm{p}<0.05$. Wilcoxon rank-sum tests were used to compare continuous variable. Chi-square tests were used to compare categorical variables. The difference between pre-operative and post-operative variables was evaluated by paired test. All analyses were conducted in STATA (Version 14.2) and R (Version 3.6.1).

\section{Results}

Patient characteristics of GERD and non-GERD groups 187 patients were enrolled and completed follow-up one year after surgery. There were 40 patients $(21 \%)$ with a confirmed diagnosis of GERD who tended to be older $(50.2 \pm 1.9$ years (GERD) $v s 44.5 \pm 1.2$ years (non-GERD), $\mathrm{p}=0.02$ ) (Tab. I). The prevalence of nasal polyposis was not statistically different between GERD and non-GERD CRS patients $(35 \%$ vs $52 \%, \mathrm{p}=0.06)$. One GERD patient was re-operated by the one-year follow-up; for the nonGERD group, five were re-operated.

\section{Pre-operative quality of life}

In univariable analyses, pre-operative total SNOT-22 scores were significantly higher in CRS patients with GERD, AR, asthma and obesity. Significance of GERD, AR and asthma was confirmed in multivariable analysis, where female gender was also independently associated with higher pre-operative scores (Tab. II). Pre-operative GERD-HRQL score was significantly higher (worse symptoms) in the GERD $v s$ non-GERD group $(16.2 \pm 0.6 v s 7.9 \pm 0.3, \mathrm{p}<0.001)$, with a moderate correlation between GERD-HRQL and total pre-operative SNOT-22 scores $(\mathrm{r}=0.39, \mathrm{p}<0.001)$. Pre-operative RSI of the GERD group was also significantly higher (worse symptoms) than the non-GERD group $(15.7 \pm 1.4$ vs $9.4 \pm 0.6, \mathrm{p}<0.001)$, and demonstrated a moderate correlation with total pre-operative SNOT-22 score $(r=0.53, p<0.001)$. 
Table I. Characteristics of GERD and Non-GERD Groups*.

\begin{tabular}{|c|c|c|c|}
\hline Variables & GERD & Non-GERD & $\mathrm{p}$-value \\
\hline \multicolumn{4}{|l|}{ Clinico-demographic factors } \\
\hline Sex (female/male) & $21 / 19$ & $63 / 84$ & 0.28 \\
\hline BMI (under/normal/over/obese) * & 0/17/15/8 & $3 / 64 / 61 / 19$ & 0.57 \\
\hline Smoking (never/previous/current) & $28 / 5 / 7$ & $107 / 15 / 25$ & 0.91 \\
\hline Allergic rhinitis (yes/no) & $22 / 18$ & $60 / 87$ & 0.11 \\
\hline Asthma (yes/no) & $4 / 36$ & 16/131 & 0.87 \\
\hline Deviated septum (yes/no) & $13 / 27$ & $61 / 86$ & 0.30 \\
\hline OSAHS (yes/no) ${ }^{*}$ & $0 / 40$ & $6 / 141$ & 0.19 \\
\hline Diabetes (yes/no) & $0 / 40$ & $3 / 144$ & 0.36 \\
\hline \multicolumn{4}{|l|}{ Outcomes } \\
\hline SNOT-22 improvement (1 year) & $-26.2 \pm 2.6$ & $-16.1 \pm 1.4$ & 0.001 \\
\hline Post-operative SNOT-22 & $17.7 \pm 2.7$ & $13.5 \pm 1.1$ & 0.25 \\
\hline Pre-operative GERD-HRQL* & $16.2 \pm 0.6$ & $7.9 \pm 0.3$ & $<0.001$ \\
\hline Pre-operative RSI ${ }^{\star}$ & $15.7 \pm 1.4$ & $9.4 \pm 0.6$ & $<0.001$ \\
\hline Pre-operative Lund-Mackay (CT)* & $7.2 \pm 1.2$ & $10.1 \pm 0.6$ & 0.01 \\
\hline Pre-operative Lund-Kennedy (endoscopy) & $6.0 \pm 0.5$ & $6.7 \pm 0.2$ & 0.23 \\
\hline Lund-Kennedy improvement (12 weeks) & $-3.2 \pm 0.6$ & $-3.2 \pm 0.2$ & 0.81 \\
\hline Post-operative Lund-Kennedy & $3.2 \pm 0.3$ & $3.6 \pm 0.2$ & 0.17 \\
\hline
\end{tabular}

For continuous factors, mean and standard error were provided. The GERD group tended to be older compared to non-GERD group. Greater pre-operative SNOT-22 score and SNOT-22 improvement were found in GERD patients vs non-GERD patients. GERD: Gastroesophageal reflux disease; SNOT-22: Sinonasal outcome test-item 22; BMI: Body mass index; Under/ normal/over/obese: Underweight/ normal weight/ overweight/ obesity; OSAHS: Obstructive sleep apnoea hypopnoea syndrome; GERD-HRQL: Gastro-oesophageal reflux diseasehealth related quality of life; RSl: Reflux symptom index; CT: Computed tomography.

Post-operative improvement on total quality of life

Of 187 patients with one-year follow up, $40(21 \%)$ were diagnosed with GERD pre-operatively. Regardless of GERD status, total SNOT-22 scores improved significantly post-FESS (Fig. 2). In both univariable and multivariable analyses, SNOT-22 scores in GERD patients were associated with significantly greater improvement than SNOT-22 scores of non-GERD patients (Tab. III). The pre-operative SNOT-22 scores were associated with post-operative SNOT-22 improvement in univariable and multivariable analysis ( $\mathrm{p}<0.001$ for each comparison). Post-operative total SNOT-22 scores were now similar between the GERD and non-GERD groups (Tab. I). Nevertheless post-operative SNOT-22 scores in the GERD group were significantly higher than the non-GERD group in CRSsNP patients $(17.8 \pm 3.3$ vs $9.8 \pm 1.0, \mathrm{p}=0.03)(\mathrm{Tab} . \mathrm{IV})$. This association between the presence of GERD and greater post-operative SNOT-22 improvement in CRSsNP patients was observed in univariable and multivariable analysis $(\mathrm{p}<0.01$ for each comparison). In contrast for CRSwNP patients, no significant differences were observed in pre-operative SNOT-22 scores or post-operative SNOT-22 scores between GERD and non-GERD groups (Tab. IV).

\section{Analyses on QOL subdomains}

Overall, the five subdomains had similar relationship in that all subdomains in both the GERD and non-GERD groups improved substantially after FESS $(p<0.001$ for each comparison; Fig. 3).

Pre-operatively, CRS patients with GERD had greater impairment in otologic/facial symptoms, psychological dysfunction and sleep dysfunction subdomains than nonGERD CRS patients (Tab. V). All three subdomains improved substantially more after FESS in the GERD ( $v s$ the non-GERD) group; however, there remained a smaller but significant difference in the GERD vs non-GERD group post-operatively for sleep dysfunction and otologic/facial symptoms. The post-operative sleep dysfunction was moderately associated with otologic/facial symptoms $(r=0.40$, $p<0.001)$. A similar trend was found in the subset of patients with CRSsNP. The post-operative otologic/facial symptoms subdomain scores were significantly higher in 
Table II. Univariable and multivariable analysis of pre-operative SNOT-22 Score*.

\begin{tabular}{|c|c|c|c|}
\hline Factor & $\begin{array}{c}\text { Score } \\
\text { Mean } \pm \text { SE }\end{array}$ & $\begin{array}{c}\text { Univariable analysis } \\
\text { p-value }\end{array}$ & $\begin{array}{c}\text { Multivariable analysis } \\
\text { p-value }\end{array}$ \\
\hline Sex (female) & $33.1 \pm 1.8$ & & \\
\hline Sex (male) & $32.3 \pm 2.0$ & 0.46 & 0.03 \\
\hline Age & - & 0.25 & 0.06 \\
\hline BMI (normal weight)* & $32.4 \pm 2.1$ & & \\
\hline BMI (underweight)* & $23.7 \pm 14.3$ & & 0.06 \\
\hline BMI (overweight)* & $30.5 \pm 1.9$ & & 0.86 \\
\hline BMI (obesity) ${ }^{*}$ & $40.5 \pm 3.8$ & 0.01 & 0.22 \\
\hline Never smoker & $33.5 \pm 1.6$ & & \\
\hline Previous smoker & $30.0 \pm 4.4$ & & 0.39 \\
\hline Current smoker & $31.0 \pm 2.8$ & 0.28 & 0.75 \\
\hline GERD* (no) & $29.6 \pm 1.4$ & & \\
\hline GERD* (yes) & $44.0 \pm 3.3$ & $<0.001$ & $<0.001$ \\
\hline Allergic rhinitis (no) & $29.0 \pm 1.6$ & & \\
\hline Allergic rhinitis (yes) & $37.6 \pm 2.2$ & $<0.001$ & $<0.001$ \\
\hline Asthma (no) & $31.2 \pm 1.3$ & & \\
\hline Asthma (yes) & $45.3 \pm 5.0$ & $<0.01$ & 0.03 \\
\hline Nasal polyposis (no) & $31.0 \pm 1.9$ & & Not included in final model \\
\hline Nasal polyposis (yes) & $34.4 \pm 1.9$ & 0.21 & \\
\hline Deviated septum (no) & $33.3 \pm 1.8$ & & \\
\hline Deviated septum (yes) & $31.8 \pm 2.0$ & 0.68 & \\
\hline $\mathrm{OSAHS}^{*}$ (no) & $32.9 \pm 1.4$ & & \\
\hline OSAHS* (yes) & $26.2 \pm 9.7$ & 0.32 & \\
\hline Diabetes (no) & $32.7 \pm 1.4$ & & \\
\hline Diabetes (yes) & $31.3 \pm 12.9$ & 0.96 & \\
\hline Hypertension (no) & $32.8 \pm 1.4$ & & \\
\hline Hypertension (yes) & $28.3 \pm 2.7$ & 0.81 & \\
\hline Alcohol use (rare) & $33.5 \pm 1.5$ & & \\
\hline Alcohol use (frequent) & $29.4 \pm 2.7$ & 0.23 & \\
\hline
\end{tabular}

SNOT-22 scores in subgroups were presented. Global p-value was used in univariable analysis. Multiple linear regression used normalised SNOT-22 score, p-value of categorical independent variables was compared to the reference category. The presence of GERD was associated with significant higher pre-operative SNOT-22 in both univariable analysis and multivariable analysis. SNOT-22: Sinonasal outcome test-item 22; BMI: Body mass index; GERD: Gastro-oesophageal reflux disease; SE: Standard error; OSAHS: Obstructive sleep apnoea hypopnoea syndrome.

CRSsNP patients with GERD than CRSsNP patients without GERD $(2.7 \pm 0.5$ vs $1.4 \pm 0.3, \mathrm{p}<0.01)$. Compared with non-GERD CRSsNP patients, the post-operative sleep dysfunction was significantly worse in CRSsNP patients with concomitant GERD $(5.4 \pm 1.2$ vs $2.1 \pm 0.3, \mathrm{p}=0.02)$. In contrast, no significant differences were found in post-operative SNOT-22 subdomains between CRSwNP patients with GERD vs without GERD.

\section{QOL in patients with improvement in specific groups}

of sinuses

We compared the prevalence of specific sinus involvement between GERD and non-GERD groups. The rate of frontal sinus involvement was significantly higher in the
non-GERD group compared to the GERD group (51\% vs $32 \%, \mathrm{p}=0.03)$. There was a difference in the proportion of patients who had anterior ethmoid sinus involvement between GERD patients and non-GERD patients (40\% vs $61 \%, \mathrm{p}=0.02$ ).

When evaluating the association between QOL and specific sinus involvement, there was a significant difference in post-operative SNOT-22 scores between CRS patients with $v s$ without maxillary sinus involvement $(15.1 \pm 1.2, \mathrm{n}=161$ vs $8.2 \pm 2.0, \mathrm{n}=26 ; \mathrm{p}<0.01)$. Associations were not found with other sinuses. The comparison of post-operative QOL in GERD vs non-GERD group was further performed in patients with/without involvement of individual sinuses. There were no significant QOL differences between GERD 


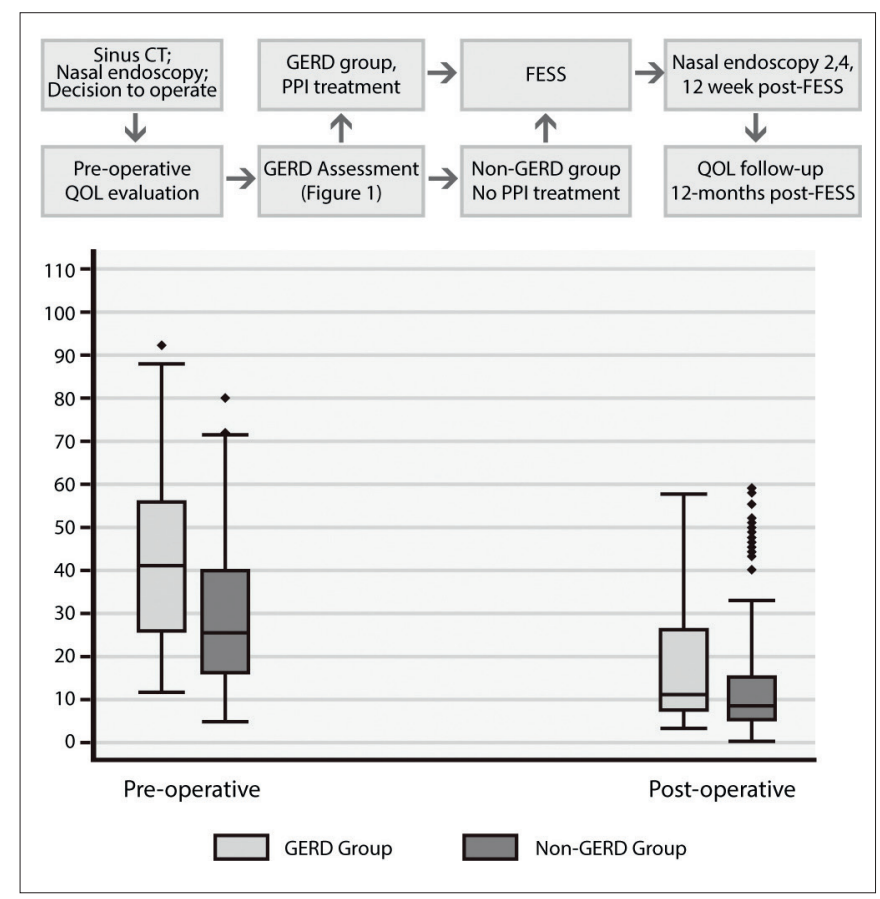

Figure 2. Boxplot of pre-operative and post-operative total SNOT-22 scores, comparing gastro-oesophageal reflux disease (GERD) and non-GERD groups. Post-operative assessments were performed at 12 months after surgery. SNOT-22: Sinonasal outcome test-item 22; CT: Computed tomography; PPIs: Proton pump inhibitors; FESS: Functional endoscopic sinus surgery; QOL: Quality of life.

and non-GERD subjects in any set of sinuses, except for a borderline association in patients who did not suffer from anterior ethmoid sinus involvement $(19.0 \pm 3.7, \mathrm{n}=24$, GERD $v s 9.7 \pm 1.4, \mathrm{n}=57$, non-GERD; $\mathrm{p}=0.04$ ).

\section{CT and endoscopic outcomes}

Compared to non-GERD patients, those with GERD showed significantly lower pre-operative total Lund-Mackay CT scores $(7.2 \pm 1.2$ vs $10.1 \pm 0.6, \mathrm{p}=0.01$; Tab. I $)$, which was confirmed by multivariable regression $(\mathrm{p}<0.01)$, after adjustment by sex, age, BMI, smoking and comorbidities. The severity grade in anterior ethmoid cells $(\mathrm{p}=0.03)$ and ostiomeatal complex $(p=0.01)$ in the Lund-Mackay system was significantly higher in non-GERD group $v s$ GERD group.

Pre-operative total Lund-Kennedy endoscopic scores were similar in CRS patients with and without GERD $(6.0 \pm 0.5$ vs $6.7 \pm 0.2, \mathrm{p}=0.23$ ). Lund-Kennedy scores were significantly decreased 12 weeks after FESS in both the GERD and non-GERD group ( $\mathrm{p}<0.001$ for each comparison). No significant differences were observed in Lund-Kennedy scores after FESS between GERD and non-GERD patients (Tab. I).

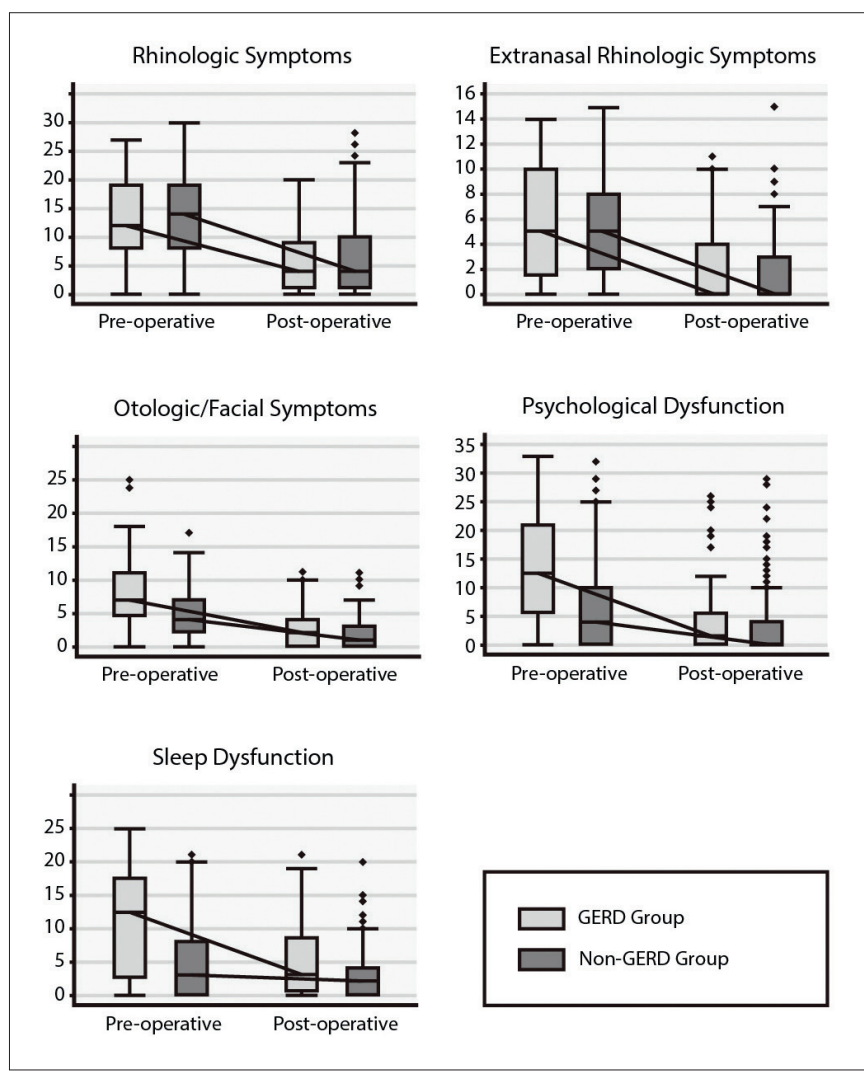

Figure 3. Boxplot of pre-operative and post-operative SNOT-22 subdomains of chronic rhinosinusitis (CRS) patients with and without gastro-oesophageal reflux disease (GERD). Post-operative assessments were performed at 12 months after surgery. SNOT-22: Sinonasal outcome test-item 22.

\section{Summary of major findings}

CRS patients with GERD experienced significantly worse pre-operative QOL compared to non-GERD CRS patients, but higher QOL improvements following FESS, resulting in no differences in overall QOL one-year post-FESS in those with $v s$ without GERD. In subdomain analyses, impairment in one-year post-FESS sleep dysfunction and otologic/facial symptoms were greater in GERD patients (compared to non-GERD patients); these greater symptoms seemed to be driven by the subset of patients who never had nasal polyps, as these differences by GERD-status were not observed in CRS patients who did have nasal polyps.

\section{Discussion}

Comparison of results with previous studies

The mechanisms of how GERD affects the sinonasal cavity remain unclear. Extra-oesophageal reflux may reach the proximal aerodigestive tract, exacerbating the symptoms of CRS. Few studies have investigated the role of GERD on outcomes of FESS and have reported mixed results. In our study, the presence of GERD was associated with worse 
Table III. Univariable and multivariable analysis of post-operative SNOT-22 improvement*.

\begin{tabular}{|c|c|c|c|}
\hline Factors & $\begin{array}{l}\text { Improvement } \\
\text { Mean } \pm \text { SE }\end{array}$ & $\begin{array}{l}\text { Univariable analysis } \\
\qquad \mathrm{p} \text {-value }\end{array}$ & $\begin{array}{c}\text { Multivariable analysis } \\
\text { p-value }\end{array}$ \\
\hline Sex (female) & $-18.2 \pm 1.9$ & & \\
\hline Sex (male) & $-18.5 \pm 1.8$ & 0.92 & 0.54 \\
\hline Age & - & 0.72 & 0.57 \\
\hline BMI (normal weight)* & $-17.4 \pm 1.2$ & & \\
\hline BMI (underweight) & $-20.0 \pm 8.1$ & & 0.69 \\
\hline BMI (overweight)* & $-18.2 \pm 1.1$ & & 0.50 \\
\hline BMI (obesity) ${ }^{*}$ & $-22.8 \pm 2.7$ & 0.21 & 0.03 \\
\hline Never smoker & $-18.7 \pm 1.5$ & & \\
\hline Previous smoker & $-11.8 \pm 3.8$ & & 0.18 \\
\hline Current smoker & $-21.0 \pm 3.2$ & 0.10 & 0.88 \\
\hline GERD* (no) & $-16.1 \pm 1.4$ & & \\
\hline GERD* (yes) & $-26.2 \pm 2.6$ & 0.001 & $<0.001$ \\
\hline OSAHS* (no) & $-19.0 \pm 1.3$ & & \\
\hline OSAHS* (yes) & $1.5 \pm 7.2$ & 0.01 & $<0.001$ \\
\hline Nasal polyposis (no) & $-18.9 \pm 1.6$ & & Not included in final model \\
\hline Nasal polyposis (yes) & $-18.3 \pm 2.0$ & 0.82 & \\
\hline Allergic rhinitis (no) & $-17.3 \pm 1.8$ & & \\
\hline Allergic rhinitis (yes) & $-19.9 \pm 1.9$ & 0.33 & \\
\hline Asthma (no) & $-18.1 \pm 1.4$ & & \\
\hline Asthma (yes) & $-20.2 \pm 3.1$ & 0.63 & \\
\hline Deviated septum (no) & $-20.0 \pm 1.6$ & & \\
\hline Deviated septum (yes) & $-15.9 \pm 2.1$ & 0.12 & \\
\hline Diabetes (no) & $-18.2 \pm 1.3$ & & \\
\hline Diabetes (yes) & $-25.0 \pm 11.9$ & 0.51 & \\
\hline Hypertension (no) & $-18.3 \pm 1.3$ & & \\
\hline Hypertension (yes) & $-20.7 \pm 4.1$ & 0.72 & \\
\hline No smoking post-surgery & $-22.1 \pm 3.4$ & & \\
\hline Smoking post-surgery & $-17.7 \pm 1.4$ & 0.23 & \\
\hline Reoperation (no) & $-18.6 \pm 1.3$ & & \\
\hline Reoperation (yes) & $-10.8 \pm 6.4$ & 0.29 & \\
\hline Alcohol use (rare) & $-18.1 \pm 3.0$ & & \\
\hline Alcohol use (frequent) & $-18.4 \pm 1.4$ & 0.94 & \\
\hline
\end{tabular}

Univariable analysis used global p-value. Multivariable analysis was conducted using multiple linear regression, $p$-value of categorical independent variables was compared to the reference category. SNOT-22: Sinonasal outcome test-item 22; BMI: Body mass index; GERD: Gastro-oesophageal reflux disease; SE: Standard error; OSAHS: Obstructive sleep apnoea hypopnoea syndrome.

baseline QOL symptoms related to CRS prior to surgery despite medical management for CRS. There was also a suggestion that CRS patients with GERD had radiologically less severe CRS at presentation, despite having worse symptoms. However, patients with GERD demonstrated greater improvements in QOL symptoms (by SNOT-22 scores) following FESS, resulting in similar overall QOL one year post-operatively.

These findings contrast those from Chambers et al., who reported that GERD was a prognostic predictor for FESS failure. In their study, $41 \%$ of patients reporting a poor re- sult from FESS had GERD compared to $20 \%$ of patients with a good outcome from the surgery; however, recall bias may be a major factor as the data was collected only retrospectively with a mean of 42.5 months post-FESS, and was based on self-report without confirmation of GERD diagnosis ${ }^{6}$. While DeConde et al. found no significant difference by GERD in SNOT-22 score improvements, the QOL of these patients was similar prior to surgery, and there were methodological issues with the study: this study used a previous recorded diagnosis of GERD obtained from the medical record or the use of anti-GERD medication 
as proxy definitions ${ }^{8}$. Although one study identified nasopharyngeal reflux as being associated with recalcitrant CRS following FESS, we found no differences in re-operative rates after initial surgery between GERD and non-GERD patients ${ }^{7}$. Furthermore, in our population, re-operation rates were very low, which may make it difficult to identify differences in re-operation rates, even if they existed. In our results, the proportion of nasal polyposis patients was not significantly different between GERD vs non-GERD group, in consistence with literature before ${ }^{18}$. Chowdhury et al. reported pre-operative QOL as a prognostic factor for post-operative outcomes in CRS patients ${ }^{19}$. Pre-operative SNOT-22 was associated with post-operative improvement of CRS-related QOL in our study.

\section{Potential mechanism of greater benefit from FESS in CRS patients with GERD}

The study hypothesis is that with maximal medical management of GERD and CRS, FESS is a good method of treating both conditions. The theory of improved peak nasal inspiratory flow (PNIF) might explain why CRS patients with GERD experienced greater improvement $v s$ non-GERD CRS patients in our study.

PNIF is an objective measure of nasal patency, correlated with QOL in post-FESS patients. Significant improvement of PNIF was found after FESS by Whitcroft et al., and post-FESS change of PNIF was strongly associated with improvement of SNOT-22 score. PNIF was also shown to be influenced by hydrochloric acid infusion, and acid infusion is traditionally regarded to have an important mechanistic role in GERD ${ }^{20}$. FESS might change PNIF in CRS patients regardless of the presence or absence of GERD, resulting in QOL improvement in CRS patients with and without GERD; however, improved PNIF may have additional secondary benefit to patients with GERD, resulting in greater improvements in QOL scores in patients with both CRS and GERD.

\section{Potential mechanism of the association between GERD} and $C R S$

The presence of GERD was related to worse pre-operative QOL among CRS patients in our study. There are several theories addressing how GERD affects the sinonasal cavity. Firstly, reflux content may have a direct effect on the nasal mucosa, where the evidence lies in the response of the nasal mucosa that is similar to the response by the oesophageal mucosa during direct contact with gastric contents: pepsin A and heat shock protein 70 expression ${ }^{21}$. Secondly, Helicobacter pylori may be involved in mechanism of CRS by promoting nasal polyp. Thirdly, dysfunction of autonomic nervous system attributed to GERD may result in reduction of PNIF, through neural reflex exists between oesophagus and paranasal sinuses via the vagus nerve. The lower LundMackay scores but greater QOL impairment in CRS patients with GERD by our study is supported by a previous work showing that GERD could impact CRS symptoms through autonomic dysfunction more than sinonasal inflammatory status alone. ${ }^{22}$ Because the prevalence of specific sinus involvement varied between GERD patients and non-GERD patients in our results, the anatomical underpinnings of individual paranasal sinuses might influence the effect of GERD on the aerodigestive tract ${ }^{23}$. Future analyses in other datasets will be necessary to answer this question.

In our study, CRS QOL was positively associated with GERD and LPR symptoms. LPR may be an intermediary mechanism between GERD and CRS, and LPR may even play a role in the development of CRS.

\section{The effects of PPIs on CRS}

Several studies found conflicting results about effects of PPIs therapy on symptoms improvement in CRS patients. ${ }^{24}$ In our study, the pre-operative CRS symptoms were worse in patients with GERD $v s$ patients without GERD prior to initiation of PPIs treatment. Low $\mathrm{pH}$ and gastric enzymes of the gastric content to upper aerodigestive tract may damage airways mucous membrane. PPIs are the most potent

Table IV. CRS QOL in CRSSNP and CRSwNP patients".

\begin{tabular}{|c|c|c|c|}
\hline Variables & GERD* & Non-GERD* & $\mathrm{p}$-value \\
\hline \multicolumn{4}{|l|}{ CRS QOL in CRSsNP patients } \\
\hline Pre-operative SNOT-22 scores* & $44.7 \pm 3.9$ & $25.9 \pm 1.8$ & $<0.001$ \\
\hline Post-operative SNOT-22 scores & $17.8 \pm 3.3$ & $9.8 \pm 1.0$ & 0.03 \\
\hline \multicolumn{4}{|l|}{ CRS QOL in CRSwNP patients } \\
\hline Post-operative SNOT-22 scores & $17.5 \pm 4.9$ & $16.4 \pm 1.8$ & 0.80 \\
\hline
\end{tabular}


Table V. SNOT-22 subdomain analysis".

\begin{tabular}{|c|c|c|c|}
\hline Subdomains & GERD & Non-GERD & $\mathrm{p}$-value \\
\hline \multicolumn{4}{|l|}{ Pre-operative SNOT-22 subdomains } \\
\hline Rhinologic symptoms & $13.1 \pm 1.2$ & $13.4 \pm 0.6$ & 0.84 \\
\hline Extranasal rhinologic symptoms & $6.0 \pm 0.8$ & $5.2 \pm 0.3$ & 0.48 \\
\hline Otologic/facial symptoms & $8.1 \pm 0.9$ & $4.8 \pm 0.3$ & $<0.001$ \\
\hline Psychological dysfunction & $13.8 \pm 1.6$ & $6.9 \pm 0.6$ & $<0.001$ \\
\hline Sleep dysfunction & $11.0 \pm 1.4$ & $5.1 \pm 0.5$ & $<0.001$ \\
\hline \multicolumn{4}{|l|}{ SNOT-22 subdomains improvement } \\
\hline Rhinologic symptoms & $-7.0 \pm 1.1$ & $-7.0 \pm 0.6$ & 0.90 \\
\hline Extranasal rhinologic symptoms & $-3.4 \pm 0.8$ & $-3.3 \pm 0.3$ & 0.94 \\
\hline Otologic/facial symptoms & $-5.5 \pm 0.7$ & $-3.1 \pm 0.3$ & 0.001 \\
\hline Psychological dysfunction & $-8.5 \pm 1.6$ & $-3.5 \pm 0.7$ & 0.001 \\
\hline Sleep dysfunction & $-6.0 \pm 1.2$ & $-2.3 \pm 0.5$ & 0.01 \\
\hline \multicolumn{4}{|c|}{ Post-operative SNOT-22 subdomains } \\
\hline Rhinologic symptoms & $6.1 \pm 1.0$ & $6.5 \pm 0.5$ & 0.83 \\
\hline Extranasal rhinologic symptoms & $2.5 \pm 0.5$ & $2.0 \pm 0.3$ & 0.43 \\
\hline Otologic/facial symptoms & $2.6 \pm 0.4$ & $1.8 \pm 0.2$ & 0.04 \\
\hline Psychological dysfunction & $5.3 \pm 1.3$ & $3.3 \pm 0.5$ & 0.14 \\
\hline Sleep dysfunction & $5.1 \pm 0.9$ & $2.8 \pm 0.3$ & 0.01 \\
\hline
\end{tabular}

Subdomain scores were presented as mean \pm standard error. P-values were generated from Wilcoxon rank-sum test. SNOT-22: Sinonasal outcome test-item 22; GERD: Gastrooesophageal reflux disease.

and most widely used group of drugs to treat reflux disease $^{24}$.

\section{GERD and CRS patients without nasal polyposis}

In our study, post-operative CRS-related QOL impairment was greater in CRSsNP patients with GERD than those without GERD, and significantly higher SNOT-22 scores were seen in the GERD group $v s$ the non-GERD group in CRSsNP patients. The different nasal mucosal molecular characteristics between CRSsNP and CRSwNP patients may indicate the greater mucosal damage in CRSsNP patients with concurrent GERD. The positive rates of pep$\sin \mathrm{A}$ in turbinate mucosa of middle concha of CRSsNP patients were higher than polyp tissue from CRSwNP patients. MUC5AC, one of several mucins, demonstrated a lower expression in the nasal mucosa of CRSsNP patients when compared with CRSwNP. Reflux has effects on mucin expression. The downregulation of MUC5AC was associated with mucosal damage ${ }^{21}$.

\section{Sleep problems remained in post-operative CRS patients with GERD}

As a significant difference in the SNOT-22 sleep dysfunction subdomain scores remained one year post-FESS in our study; this difference may be explained through a bidirectional relationship between GERD and sleep problems. Nocturnal reflux may lead to sleep deprivation, and sleep deprivation could exacerbate GERD through enhancing intra-oesophageal stimulating perception. Otologic/facial pain and sleep dysfunction scores have been correlated in CRS patients in another study, a similar relationship was observed in our study ${ }^{25}$.

This study has several strengths. Firstly, this was a prospective analysis with broad eligibility criteria useful as real-world evidence. Secondly, we completed extensive study procedures to confirm GERD diagnosis in our patients. Thirdly, validated disease-specific instruments were used to assess and analyse CRS symptom-outcomes. However, there are several limitations: (i) our patients did not receive long term PPIs prior to FESS and we were not able to collect serial QOL assessment after PPIs initiation. Thus, we could not conclusively differentiate between improvements in QOL due to PPIs therapy for GERD vs FESS treatment for CRS; (ii) specific GERD questionnaires were not administered at the 12-month follow-up; (iii) information of endotypes was unavailable in this study (e.g. eosinophil level in CRS mucosa); accordingly, we were unable to explain our findings according to the types of underlying inflammatory response (type-2 and non-type-2); (iv) there is limited generalisability in this single-institution study.

\section{Conclusions}

In summary, we conducted a prospective study on the relationship between GERD and CRS related QOL before 
and after FESS. We found greater pre-operative CRS and GERD symptoms in the subset of CRS patients diagnosed concurrently with GERD. After FESS, these overall symptoms significantly improved more in CRS patients with GERD $v s$ those without GERD, but a small difference remained post-operatively in several subdomains of CRS even at one-year follow-up, mostly driven by the subgroup of CRS patients who did not have nasal polyps.

Future research is needed to assess serial reflux related QOL outcomes in CRS patients with GERD after FESS, the longterm effect of PPIs on both CRS and GERD symptoms, and the specific conditions that result in FESS failure that required a second operation in CRS patients with GERD.

\section{Acknowledgements}

The authors thank staff in the West China Biobanks of Department of Clinical Research Management, the nurses involved in the care of these patients in West China Hospital, the staff in Geoffrey Liu's office in Princess Margaret Cancer Centre, Mr. Lin Zhiwei for his edit of figures, and the patients themselves for their contribution.

\section{References}

1 Fokkens WJ, Lund VJ, Hopkins C, et al. European Position Paper on rhinosinusitis and nasal polyps 2020. Rhinology 2020;58:1-464. https://doi.org/10.4193/Rhin20.600

2 Liu Z, Chen J. Chinese Society of Allergy and Chinese Society of Otorhinolaryngology-Head and Neck Surgery Guideline for Chronic Rhinosinusitis. Allergy Asthma Immunol Res 2020;12:176-237. https://doi.org/10.4168/aair.2020.12.2.176

3 Chung SD, Hung SH, Lin HC, et al. Health care service utilization among patients with chronic rhinosinusitis: a population-based study. Laryngoscope 2014;124:1285-1289. https://doi.org/10.1002/ lary. 24500

4 Tan VP, Wong BC, Wong WM, et al. Gastroesophageal reflux disease: cross-sectional study demonstrating rising prevalence in a Chinese population. J Clin Gastroenterol 2016;50:e1-7. https://doi. org $/ 10.1097 / \mathrm{mcg} .0000000000000304$

5 Leason SR, Barham HP, Oakley G, et al. Association of gastro-oesophageal reflux and chronic rhinosinusitis: systematic review and meta-analysis. Rhinology 2017;55:3-16. https://doi.org/10.4193/ Rhin 16.177

6 Chambers DW, Davis WE, Cook PR, et al. Long-term outcome analysis of functional endoscopic sinus surgery: correlation of symptoms with endoscopic examination findings and potential prognostic variables. Laryngoscope 1997;107:504-510. https://doi. org/10.1097/00005537-199704000-00014

7 DelGaudio JM. Direct nasopharyngeal reflux of gastric acid is a contributing factor in refractory chronic rhinosinusitis. Laryngoscope 2005;115:946-957. https://doi.org/10.1097/01. mlg.0000163751.00885.63

8 DeConde AS, Mace JC, Smith TL. The impact of comorbid gastroesophageal reflux disease on endoscopic sinus surgery quality-of-life outcomes. Int Forum Allergy Rhinol 2014;4:663-669. https://doi. org/10.1002/alr.21333
9 Lin Y, Li Y, Liang M, et al. Acid exposure time $>6 \%$ might not improve the therapeutic outcome in chinese gastroesophageal reflux disease patients. J Neurogastroenterol Motil 2021;27:55-62. https://doi. org/10.5056/jnm19219

10 Katz PO, Gerson LB, Vela MF. Guidelines for the diagnosis and management of gastroesophageal reflux disease. Am J Gastroenterol 2013;108:308-328. https://doi.org/10.1038/ajg.2012.444

11 Padia R, Curtin K, Peterson K, et al. Eosinophilic esophagitis strongly linked to chronic rhinosinusitis. Laryngoscope 2016;126:1279-1283. https://doi.org/10.1002/lary.25798

12 Bateman ED, Hurd SS, Barnes PJ, et al. Global strategy for asthma management and prevention: GINA executive summary. Eur Respir J 2008;31:143-178. https://doi.org/10.1183/09031936.00138707

13 Brożek JL, Bousquet J, Agache I, et al. Allergic Rhinitis and its Impact on Asthma (ARIA) guidelines-2016 revision. J Allergy Clin Immunol 2017;140:950-958. https://doi.org/10.1016/j.jaci.2017.03.050

14 Stammberger H, Posawetz W. Functional endoscopic sinus surgery. Concept, indications and results of the Messerklinger technique. Eur Arch Otorhinolaryngol 1990;247:63-76. https://doi.org/10.1007/ bf00183169

15 Kennedy DW, Zinreich SJ, Shaalan H, et al. Endoscopic middle meatal antrostomy: theory, technique, and patency. Laryngoscope 1987;97:1-9. https://doi.org/10.1288/00005537-198708002-00001

16 Kastl KG, Reichert M, Scheithauer MO, et al. Patient comfort following FESS and Nasopore ${ }^{\circledR}$ packing, a double blind, prospective, randomized trial. Rhinology 2014;52:60-65. https://doi.org/10.4193/ Rhin 13.020

17 DeConde AS, Bodner TE, Mace JC, et al. Response shift in quality of life after endoscopic sinus surgery for chronic rhinosinusitis. JAMA Otolaryngol Head Neck Surg 2014;140:712-719. https://doi. org/10.1001/jamaoto.2014.1045

18 Mahdavinia M, Bishehsari F, Hayat W, et al. Prevalence of allergic rhinitis and asthma in patients with chronic rhinosinusitis and gastroesophageal reflux disease. Ann Allergy Asthma Immunol 2016;117:158-162.e1. https://doi.org/10.1016/j.anai.2016.05.018

19 Chowdhury NI, Turner JH, Dorminy C, et al. Preoperative qualityof-life measures predict acute postoperative pain in endoscopic sinus surgery. Laryngoscope 2019;129:1274-1279. https://doi.org/10.1002/ lary. 27763

20 Chen CL, Orr WC. Autonomic responses to heartburn induced by esophageal acid infusion. J Gastroenterol Hepatol 2004;19:922-926. https://doi.org/10.1111/j.1440-1746.2004.03397.x

21 Ren JJ, Zhao Y, Wang J, et al. Pepsin A as a marker of laryngopharyngeal reflux detected in chronic rhinosinusitis patients. Otolaryngol Head Neck Surg 2017;156:893-900. https://doi. org/10.1177/0194599817697055

22 Chen WC, Chang YT, Chen SF, et al. The symptom burden of autonomic dysfunction is positively associated with chronic rhinosinusitis status. Rhinology 2018;56:227-233. https://doi.org/10.4193/ Rhin18.005

23 Lipan MJ, Reidenberg JS, Laitman JT. Anatomy of reflux: a growing health problem affecting structures of the head and neck. Anat Rec B New Anat 2006;289:261-270. https://doi.org/10.1002/ar.b.20120

24 Anzic SA, Turkalj M, Zupan A, et al. Eight weeks of omeprazole 20 $\mathrm{mg}$ significantly reduces both laryngopharyngeal reflux and comorbid chronic rhinosinusitis signs and symptoms: randomised, doubleblind, placebo-controlled trial. Clin Otolaryngol 2018;43:496-501. https://doi.org/10.1111/coa.13005

25 Cox DR, Ashby S, Mace JC, et al. The pain-depression dyad and the association with sleep dysfunction in chronic rhinosinusitis. Int Forum Allergy Rhinol 2017;7:56-63. https://doi.org/10.1002/alr.21843 